\title{
Seismological Properties of Evolutionary Delta Scuti Models Using OPAL Opacities
}

\author{
Joyce A. Guzik and Paul A. Bradley \\ Los Alamos National Laboratory, Los Alamos, NM 87545
}

\begin{abstract}
We present the first results of our project of calculating the evolutionary and seismological properties of $\delta$ Scuti models using Livermore OPAL opacities. We calculate the radial and low-degree nonradial nonadiabatic pulsation frequencies for a 1.8 and $2.0 \mathrm{M}_{\odot}$ model. Our radial first overtone/fundamental mode period ratios are $\sim 0.772$, which agree well with observed period ratios. We find a dense spectrum of unstable nonradial modes including both low order $g$-type modes and low to intermediate order $p$-type modes, confirming that we need a selection mechanism allowing only some modes to grow to observable amplitudes.
\end{abstract}

\section{Introduction}

Considering the potential for detecting many simultaneous oscillation frequencies in individual $\delta$ Scuti stars through multi-site observing campaigns and satellite observations, we thought it timely to calculate the evolution and pulsation frequencies for a grid of $\delta$ Scuti models with modern opacity and equation of state (EOS) data for asteroseismology of $\delta$ Scuti stars. Here, we present preliminary evolution and pulsation results for $\delta$ Scuti models using the Livermore OPAL opacities (Rogers \& Iglesias 1992). We use our evolution and pulsation codes that give excellent results for solar evolutionary and helioseismic models (Guzik \& Cox 1993).

\section{Evolution and pulsation results}

Figure 1 shows the evolution tracks of $1.6,1.8$, and $2.0 \mathrm{M}_{\odot}$ stellar models using the $\mathrm{Y}=0.28$, and $\mathrm{Z}=0.02$ Livermore OPAL opacities and Alexander \& Fergusen (1994) low-temperature opacities. For this work, we use the Eggleton et al. (1973) equation of state with Coulomb corrections. For comparison, we show a $1.8 \mathrm{M}_{\odot}$ evolution track (Fig. 1) for models with the old Los Alamos (Cox \& Stewart 1965) opacities. The evolution tracks of models using the OPAL opacities are significantly cooler and less luminous for a given stellar mass than the tracks using the old opacities.

We analyze the linear radial and nonradial nonadiabatic periods and growth rates for a $1.8 \mathrm{M}_{\odot}$ model with $\mathrm{T}_{\text {eff }}=7640 \mathrm{~K}$, and a $2.0 \mathrm{M}_{\odot}$ model with $\mathrm{T}_{\text {eff }}=$ $6990 \mathrm{~K}$ (marked by triangles in Fig. 1 ). The $1.8 \mathrm{M}_{\odot}$ model burns hydrogen in a convective core of mass $\sim 0.2 \mathrm{M}_{\odot}$, while the $2.0 \mathrm{M}_{\odot}$ model has exhausted core hydrogen. In each model, the radial fundamental and first seven overtone modes 


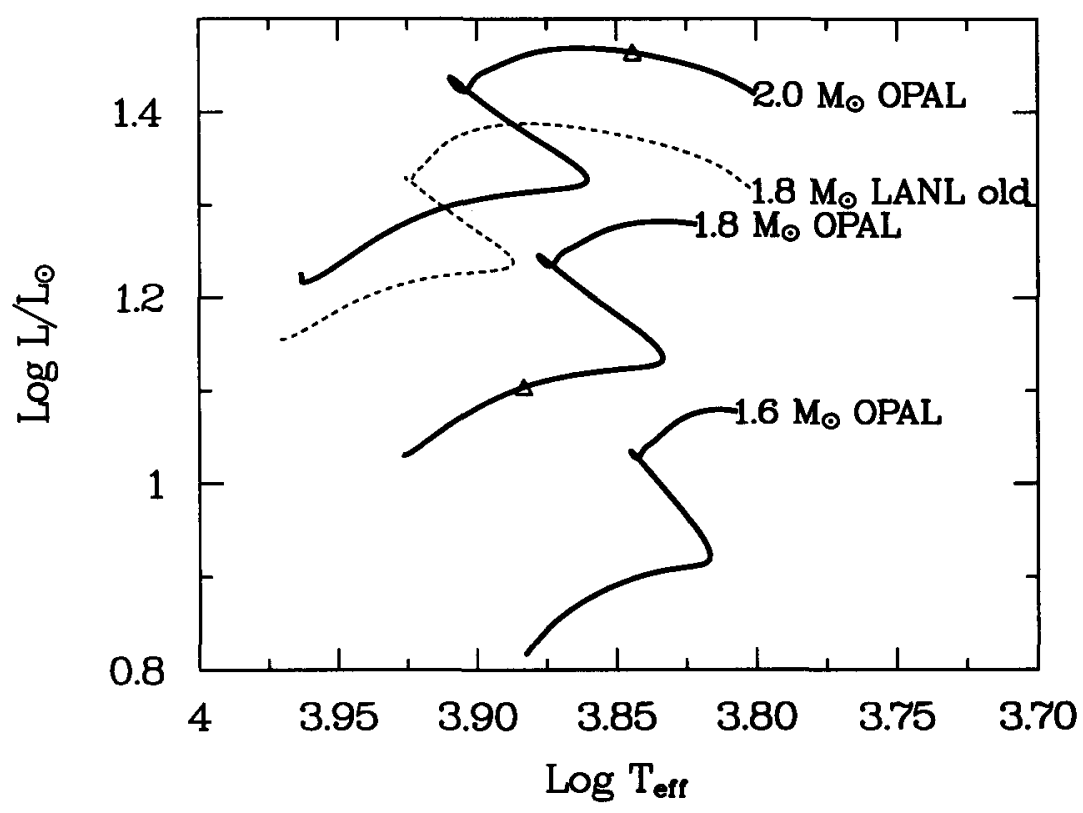

Figure 1. Evolution tracks of stellar models using the OPAL opacities (solid lines), and hotter, more luminous models using old Los Alamos opacities (dashed line).

are unstable, with the 5th and 6 th overtones being the most unstable. Our first overtone/fundamental mode period ratios are $\sim 0.772$, in excellent agreement with the observed period ratio of 0.773 (Breger, 1993).

We also find a dense spectrum of unstable low-degree $p$ - and $g$-type nonradial modes. Since there are fewer modes observed than predicted to be unstable in $\delta$ Scuti stars, either these modes are present at observationally undetectable amplitudes, or some as yet unexplained mode selection mechanism is operating.

We thank F. Swenson for providing the opacity interpolation routines, and M. Breger, J. Nemec, J. Christensen-Dalsgaard, and A.N. Cox for useful discussions. This research is supported by a NASA Astrophysics Theory Program grant and a LANL LDRD grant.

\section{References}

Alexander, D.R., \& Ferguson, J.W. 1994, ApJ, 437, 879

Breger, M., 1993, in IAU Colloq. 139, ed. J. Nemec \& J.M. Matthews, (Cambridge Univ. Press), 135

Cox, A.N., \& Stewart, J.N. 1965, ApJS, 11, 22

Eggleton, P.P., Faulkner, J., \& Flannery, B.P. 1973, A\&A, 23, 325

Guzik, J.A., \& Cox, A.N. 1993, ApJ, 411, 394

Rogers, F.J., \& Iglesias, C.A. 1992, ApJ, 401, 361 